\title{
A miR-137-related biological pathway of risk for Schizophrenia is associated with human brain emotion processing
}

Authors: G. Pergola ${ }^{1,2, \dagger},{ }^{*}$, A. Rampino ${ }^{1,3, \dagger}$, P. Di Carlo ${ }^{1,2}$, A. Marakhovskaia ${ }^{4}$, T. Quarto ${ }^{1}$, L. Fazio $^{1,5}$,

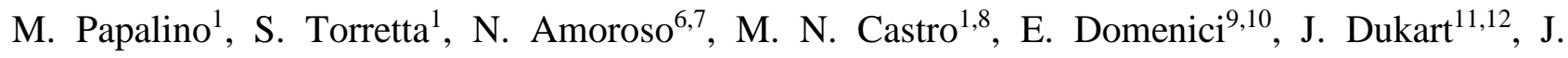
Khlghatyan $^{4,13}$, A. Monaco ${ }^{7}$, T. Popolizio ${ }^{5}$, R. Romano ${ }^{1}$, L. Sportelli ${ }^{1,2}$, H. Zunuer ${ }^{1}$, G. Blasi ${ }^{1,3}$, J.M. Beaulieu $^{4}$, A. Bertolino ${ }^{1,3, *}$

\section{Affiliations:}

${ }^{1}$ Group of Psychiatric Neuroscience, Department of Basic Medical Sciences, Neuroscience and Sense Organs, University of Bari Aldo Moro, Bari, Italy

${ }^{2}$ Lieber Institute for Brain Development, Johns Hopkins Medical Campus, Baltimore, MD (USA)

${ }^{3}$ Azienda Ospedaliero-Universitaria Consorziale Policlinico, Bari, Italy

${ }^{4}$ Department of Pharmacology, University of Toronto, Ontario, Canada

${ }^{5}$ IRCCS Casa Sollievo della Sofferenza, San Giovanni Rotondo (FG), Italy

${ }^{6}$ Dipartimento di Farmacia-Scienze del Farmaco, Università degli Studi di Bari “Aldo Moro”, Via E.

Orabona 4, I-70126 Bari, Italy

${ }^{7}$ Istituto Nazionale di Fisica Nucleare (INFN), Sezione di Bari, Via A. Orabona 4, 70125 Bari, Italy

${ }^{8}$ Consejo Nacional de Investigaciones Científicas y Técnicas (CONICET) and Grupo de Investigación en Neurociencias Aplicadas a las Alteraciones de la Conducta (Grupo INAAC), Instituto de Neurociencias FLENI-CONICET, Argentina

${ }^{9}$ Department of Cellular, Computational and Integrative Biology (CIBIO), University of Trento, Trento, Italy

${ }^{10}$ Fondazione The Microsoft Research University of Trento, Centre for Computational and Systems Biology (COSBI), Rovereto, Italy 
${ }^{11}$ Institute of Neuroscience and Medicine, Brain \& Behaviour (INM-7), Research Centre Jülich, 52428 Jülich, Germany

${ }^{12}$ Institute of Systems Neuroscience, Medical Faculty, Heinrich Heine University Düsseldorf, 40223 Düsseldorf, Germany

${ }^{13}$ Department of Neuroscience, Novartis Institutes for Biomedical Research, 250 Massachusetts, Cambridge, MA 02139, USA

${ }^{\dagger}$ GP and AR contributed equally to this work

*Please address correspondence to:

Alessandro Bertolino, MD, $\mathrm{PhD}$

Piazza G Cesare, 11

70124 Bari, Italy

Alessandro.Bertolino@uniba.it

Giulio Pergola, PhD

Piazza G Cesare, 11

70124 Bari, Italy

Giulio.Pergola@uniba.it

Number of words:

Abstract 148/150

Article 4,466 /15,000

Tables-Figures 5/6

References 79/40 


\title{
A miR-137-related biological pathway of risk for Schizophrenia is associated with human brain emotion processing
}

\begin{abstract}
:
Genome-Wide-Association studies have involved miR-137 in schizophrenia. However, the biology underlying this statistical evidence is unclear. Statistical polygenic risk for schizophrenia is associated with working memory, while other biological evidence involves miR-137 in emotion processing. We investigated the function of miR-137 target schizophrenia risk genes in humans.

We identified a prefrontal co-expression pathway of schizophrenia-associated miR-137 targets and validated the association with miR-137 expression in neuroblastoma cells. Alleles predicting greater co-expression of this pathway were associated with greater prefrontal activation during emotion processing in two independent cohorts of healthy volunteers $\left(\mathrm{N}_{1}=222 ; \mathrm{N}_{2}=136\right)$. Statistical polygenic risk for schizophrenia was instead associated with prefrontal activation during working memory.

A co-expression pathway links miR-137 and its target genes to emotion processing and risk for schizophrenia. Low prefrontal miR-137 expression may be related with SCZ risk via increased expression of target risk genes, itself associated with increased prefrontal activation during emotion processing.
\end{abstract}




\section{Introduction}

Inter-individual variation in traits with complex heritability, like psychiatric disorders, is associated with polygenic inheritance. As genes do not code for diseases, polygenic risk for psychiatric disorders like Schizophrenia (SCZ) and Autism Spectrum Disorders is likely enacted via molecular pathways affecting neurophysiological phenotypes (1-3). Accordingly, Polygenic Risk Scores cumulating the statistical effects of genetic variants associated with SCZ are associated with neural activity during working memory (4-6) and emotion processing $(7,8)$. This evidence indicates that statistical measures of genetic risk for SCZ are related with normal variation in brain features, including functional activity during cognitive and emotion processing as a possible mechanism linking risk to behavior. However, Polygenic Risk Scores do not provide information about the molecular pathways involved in the association of SCZ positive variants with system-level phenotypes. The purpose of whole-genome Polygenic Risk Scores lumping all variants together is to collect signal from all biological pathways involved. Understanding the mechanisms behind SCZ biology requires approaches integrating biological information in the score computation.

As many of the SCZ risk variants are non-coding and may control gene expression (9-11), coexpression regulation represents a potential mechanism to parse statistical summaries of genetic risk into biologically meaningful pathways. Importantly, several studies have found that SCZ risk genes are significantly co-expressed (12-14). PsychENCODE and other studies have suggested that biological networks gather converging effects of many genetic variants affecting neurophysiological and clinical phenotypes via expression quantitative trait loci $(11,15-17)$.

Candidate master regulators linking risk variants with gene expression include micro-RNAs targeting SCZ risk genes (18). Of these, miR-137 is probably the most biologically plausible candidate, potentially linking co-expression regulation with SCZ risk factors. Indeed, miR-137 genetic variation is associated with SCZ (19), with miR-137 brain transcription levels, with interindividual variability in SCZ clinical measures, and with prefrontal cortex activity and connectivity 
in healthy controls (20). The risk allele of rs1625579, a miR-137 single nucleotide polymorphism (SNP) associated with SCZ, is associated with lower miR-137 expression in the dorsolateral prefrontal cortex $(21)$, with working memory-related prefrontal activity $(22,23)$ and with activity and connectivity of the emotion processing brain network $(24,25)$. Taken together, these studies suggest that miR-137-related risk for SCZ is relevant to brain function during working memory and emotion processing, possibly via pleiotropic effects on SCZ phenotypes.

MiR-137 regulates the expression of many genes (26), therefore one possible mechanism for its effects in SCZ is co-expression regulation. Accordingly, two studies parsed the polygenic effects of miR-137 on task-based brain activation.

Potkin and coworkers (27) reported that variants associated with brain activity during WM are located in loci co-expressed with SCZ risk genes. Consistent with the role of miR-137 in orchestrating working memory gene co-expression, miR-137 target genes harbored variants associated with brain activity during working memory. However, there was no direct link between miR-137 targets and risk-related gene co-expression in the human brain.

Cosgrove and coworkers (28) parsed the SCZ-Polygenic Risk Score by including only miR137 targets and found that it was associated with brain activity during working memory. They failed to identify significant effects of the Polygenic Risk Score in 83 participants performing an emotion processing task. While these studies are in agreement on the relationship between miR-137 targets, SCZ risk, prefrontal cortex, and working memory, they do not support a functional role of miR-137 in emotion processing. This evidence suggests that miR-137 is related with SCZ via pathways common to working memory, but it is worth reiterating that Polygenic Risk Scores do not provide biological information. The question thus stands, whether miR-137 is linked with working memory per se, or whether these findings instead depend on weighting genetic variants by SCZ risk. 
At variance with the evidence derived from these approaches, molecular evidence independent of SCZ implicates miR-137 in emotion processing. MiR-137 is expressed in the amygdala more than in the prefrontal cortex (21), and its expression is upregulated by the metabotropic glutamate-receptor-5 (mGluR5); in turn, miR-137 contributes to mGluR5 function in the emotion processing brain network $(20,29)$. The lacking association between miR-137 and emotion processing in studies employing polygenic approaches may be attributed to statistical power limitations of earlier studies. Alternatively, the polygenic approaches used may have diluted the effect of risk pathways affecting emotion processing with unrelated signal, e.g., because only a subset of the SCZ loci targeted by miR-137 is associated with emotion processing. We thus hypothesized that 1) miR-137 target genes are co-expressed in the human brain with SCZ risk genes, and 2) are associated with functional brain activity during emotion processing.

Therefore, we investigated whether: i) miR-137 target genes are co-expressed in the human prefrontal cortex and how they relate with SCZ risk; ii) co-expressed miR-137 targets are related with working memory or emotion processing brain activity in humans based on biologically stratified genetic scores, and which genes are involved; iii) SCZ Polygenic Risk Scores combining variants harbored in miR-137 target genes based on SCZ risk stratification predict working memory or emotion processing brain activity in larger cohorts than those previously reported. Figure 1 reports a synopsis of the study.

We identified co-expression modules enriched for miR-137 targets in human post-mortem prefrontal cortex and validated the modules in neuroblastoma cells. We linked post-mortem coexpression networks with system-level phenotypes via co-expression quantitative trait loci (coeQTLs (30-32)), i.e., SNPs associated with gene co-expression which were combined into a Polygenic Co-expression Index (PCI $\left.\mathrm{PiR}_{\text {-137 }}\right)$ used to test further associations. The $\mathrm{PCI}_{\mathrm{miR}-137}$ indexes the predicted expression of a subset of miR-137 targets associated with SCZ risk that are co-expressed in the prefrontal cortex. Therefore, this index identifies a miR-137 related biological pathway of risk. 
Additionally, we computed a previously reported Polygenic Risk Score parsed to include variants

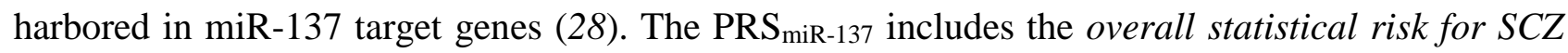
harbored in miR-137 target genes, weighted by their GWAS-derived effect size. Therefore, it is an index of miR-137 related statistical measure of genetic risk. Both $\mathrm{PCI}_{\mathrm{miR}-137}$ and $\mathrm{PRS}_{\mathrm{miR}-137}$ were tested as predictors of both working memory and emotion processing activation in the prefrontal cortex assessed via fMRI. 


\section{Results}

Our gene co-expression network included 51 modules, plus the grey module of 3,018 nonclustered genes. Permutation tests revealed that gene-gene relationships in 28 out of 51 modules were preserved in three replication datasets $\left(\mathrm{p}<\alpha_{\text {Bonferroni }}=.00033\right.$; Figure $\left.S 1\right)$. Gene loadings on the module expression were concordant across all replication datasets (binomial $\mathrm{p}$-values, all $\mathrm{p}<10^{-12}$; Figure $\mathrm{S} 2$ ), suggesting that the replications identified gene-gene relationships in the same direction as the discovery set. Four of these 28 modules were enriched for miR-137 target genes $\left(\mathrm{p}<\alpha_{\mathrm{Bonferroni}}=0.05 /(28 \times 4)=.00045\right.$; Table S3). Out of these four, only Darkorange was significantly enriched for genes in the SCZ risk loci ( $p=.0052$ with $\alpha_{\text {Bonferroni }}=0.05 / 4=0.0125$; nine genes in nine risk loci, see Table 1; Table S4 reports the list of Darkorange genes). Using a different method to quantify SCZ association, we found that genetic variants harbored within Darkorange genes were associated with greater SCZ risk compared to all remaining modules (MAGMA (33); p=.0012 with $\alpha_{\text {Bonferroni }}=0.05 / 28=0.0018$; Figure 2). The module eigengene, i.e., the first principal component of Darkorange gene expression, explained the majority (54\%) of Darkorange expression variance. Overall, the over-representation of genes and variants in the risk loci suggested that a component of the genetic risk for SCZ converged into Darkorange.

Darkorange is enriched for neuronal markers and gene ontologies relevant to schizophrenia.

Darkorange was comprised of 173 genes (156 protein-coding). Darkorange presented marker gene expression characteristic of glutamatergic and GABAergic neurons (34) (Figure S3) and was functionally enriched for biological processes relevant to SCZ (Figure 2), including synaptic signaling (GO:0099536, 19 genes, fold enrichment $\left.=5.8, \mathrm{p}_{\text {corrected }}=8.37 \times 10^{-6}\right)$ and nervous system development (GO:0007399, 41 genes, fold enrichment $=2.4$, $\left.\mathrm{p}_{\text {corrected }}=6.39 \times 10^{-4}\right)$. No chromosomal locus was significantly over-represented in Darkorange. Specific Expression Analysis revealed that 
Darkorange genes are preferentially expressed in the PFC during young adulthood $\left(\mathrm{p}_{\text {corrected }}=1.7 \times 10^{-}\right.$

5, [pSI<.01]), with a pattern closely matching published findings on SCZ genes (35) (Figure S4). Darkorange was not enriched for TWAS (all p>.05).

Darkorange topology links schizophrenia risk genes with miR-137 targets

Brainspan data confirmed that miR-137 expression was more correlated with miR-137 targets than non-targets (Wilcoxon rank-sum test, $\mathrm{p}=.044$ ). Specifically, correlations were more negative for miR-137 targets (on average, $r=-.61$ ) than for non-targets (average $r=-.47$ ). These results indicate that the genes identified above as being co-expressed with miR-137 are indeed more associated than others with miR-137 expression.

Within Darkorange, the analysis of intramodular connectivity showed that miR-137 targets were more central, i.e., more strongly connected with the rest of the module, than non-targets (Wilcoxon rank-sum tests, $\mathrm{p}=0.0093$ ). Eight out of nine SCZ risk genes (Figure 2) had higher correlations with miR-137 targets than non-targets (all $\mathrm{p}<.05$ ); gene $K C N V 1$ reached marginal significance $(\mathrm{p}=.066)$. These results indicate that miR-137 targets are connected to SCZ risk genes above chance level; SCZ risk genes are more related with targets than with non-targets within Darkorange.

Darkorange is enriched for genes modulated by miR-137

To assess directly the contribution of miR-137 to the regulation of Darkorange, we used a cellular system allowing for a titration of miR-137 expression. Plasmid transfections were used to overexpress (OE) miR-137 in a Neuro2A (N2A) neuroblastoma cells. A CRISPR/Cas9 approach (Figure S5) was also developed to disrupt (KO) miR-137 expression in these same cells. Evaluation 
of miR-137 expression using quantitative PCR confirmed the efficacy of the KO as well as OE procedures (Table S5). We quantified the effect of various levels of miR-137 expression on genomewide gene expression using microarray analysis (Supplemental Information [SI]-1.3).

We identified 580 dose-dependently expressed genes (DDEGs) showing linear covariation with miR-137 across the transcriptome ( $\left.\mathrm{q}_{\mathrm{FDR}}<.05\right)$. Darkorange included 13 DDEGs out of 141 genes expressed in neuroblastoma cells, a significant proportion ( $\mathrm{p}=.011$; Figure 2; Figure S6). We assessed whether any other replicated modules were significantly enriched as a negative control. Only few modules over-represented miR-137 DDEGs (Figure 2), consistent with the human post-mortem data and supporting the relative specificity of Darkorange for miR-137 targets.

The Polygenic Co-expression Index combines reproducible associations of SNPs with Darkorange.

We translated Darkorange gene co-expression into the $\mathrm{PCI}_{\mathrm{miR}-137}$ via the association of genetic variants with the module eigengene. SNP weights are available in Table S6. PCIs including between 7 and 15 SNPs were replicated (BRAINEAC: one-tailed $\mathrm{p}<.05$, Table S6). Table 2 includes annotations of the first 15 SNPs. We adopted an inclusive approach to collect signal from multiple module genes and therefore tested the PCI $\mathrm{I}_{\text {miR-137 }}$ including 15 SNPs in the neuroimaging sample.

Neuroimaging results confirm the association of polygenic risk for schizophrenia with working memory but reveal an association of miR-137 related co-expression with emotion processing.

Table S7 reports complete fMRI statistics. During the emotion processing task, the linear term of the PCImiR-137 correlated positively with BOLD activity in a right prefrontal cluster including Brodmann areas 8, 9, and 46 within the discovery sample (peak Z=5.1; pFwE=.001; 97 voxels; MNI coordinates $\mathrm{x}=50 ; \mathrm{y}=12 ; \mathrm{z}=42$; Figure 3 ). In the replication sample, the effect was in the same 
direction and significant in BA9 (peak $\mathrm{Z}=3.5$; $\mathrm{p}_{\mathrm{sVC}}=.007 ; 42$ voxels; MNI coordinates $\mathrm{x}=46 ; \mathrm{y}=12$; $\mathrm{z}=31$ ). Therefore, greater Darkorange predicted co-expression was associated with greater prefrontal activity during emotion processing. Greater $\mathrm{PCI}_{\mathrm{miR}-137}$ was also associated with lower prefrontalamygdala connectivity during emotion processing $\left(\mathrm{p}_{\mathrm{svC}}<.05\right.$; Figure $\left.\mathrm{S} 7\right)$. We found no effect of $\mathrm{PRS}_{\mathrm{miR}-137}$ on emotion processing.

Conversely, we found no significant effect of the PCI $\mathrm{I}_{\mathrm{miR}-137}$ on working memory-related activity. Instead, PRS $\mathrm{miR}_{-137}$ computed with two of the three $\mathrm{SCZ}$ association thresholds $(\mathrm{p}<.05$ and $\mathrm{p}<.5)$ correlated positively with working memory activity in a right prefrontal cluster including BA8 (SNP association $\mathrm{p}<.05$ threshold: peak $\mathrm{Z}=4.5 ; \mathrm{p}_{\mathrm{FWE}}=.008 ; 37$ voxels; $\mathrm{MNI}$ coordinates $\mathrm{x}=27 ; \mathrm{y}=27$; $\mathrm{Z}=58$ ). This effect was specific for $\mathrm{PRS}_{\mathrm{miR}-137}$ and was not observed when using SCZ risk variants not harbored within miR-137 targets (SI-2). Further analyses suggested that statistical power was not an issue for the $\mathrm{PCI}_{\mathrm{miR}-137} \mathrm{n}$-back analysis and removing Darkorange genes from the $\mathrm{PRS}_{\mathrm{miR}-137}$ did not affect results (SI-2). Additionally, to establish the specificity of the imaging associations, we explored ththe sameis association with PCIs derived from other modules enriched for SCZ risk genes (Black, Blue, and Lightcyan; see Figure 2) as a negative control. To compute these scores, we used the top 15 independent SNPs within the module genes like we did for the Darkorange PCI. We found no significant association with the n-back task and no replicated association with the Faces task (data available on request). 


\section{Discussion}

We hypothesized that miR-137 regulates the co-expression of SCZ risk genes in the human prefrontal cortex and evaluated the involvement of miR-137 targets in working memory and emotion processing. Indeed, we found that miR-137 targets are co-expressed in at least four reproducible coexpression modules. Of these, only Darkorange shows over-representation of SCZ genes and has a link with miR-137 supported by in vitro evidence. SNAP91, CACNB2, ATP2A2, BCL11B, GRIN2A, and NLGN4X genes appear at the intersection between miR-137-associated co-expression and SCZ risk. Gene Ontology analysis links Darkorange genes with Neurodevelopment and Synaptic Signaling, two biological functions implicated in SCZ. When we translated miR-137 effects on its targets into two polygenic indices respectively reflecting co-expression of a subset of SCZ risk genes targeted by miR-137 (PCI $\left.\mathrm{miR}_{137}\right)$ or the whole-genome association with SCZ risk (PRS $\left.\mathrm{miR}_{137}\right)$, we obtained differential neuroimaging readouts in neurotypical individuals. We replicated prior evidence that PRS $\mathrm{miR}_{-137}$ is associated with prefrontal activity during working memory, confirming that SCZweighted genetic score associate miR-137 with working memory. Instead, genetic scores based on co-expression tell a different story: greater $P C I_{m i R-137}$ derived from a subset of miR-137 targets coexpressed in the human PFC is associated with greater prefrontal activity and lower prefrontalamygdala coupling during emotion processing, but is not related with working memory. None of the three modules enriched for miR-137 but not enriched for SCZ showed an association with working memory. Taken together, this evidence shows that miR-137 targets are involved both in working memory and emotion processing SCZ phenotypes, but a subset of miR-137 potentially co-regulated genes appears more closely associated with emotion processing.

Our approach has two advantages: first, it incorporates SCZ GWAS information in the module prioritization, but also includes co-expression information - whereas the Polygenic Risk Score only includes GWAS information; second, it parses miR-137 targets into sets of co-expressed genes, whereas the Polygenic Risk Score computed with all miR-137 targets cumulates statistical effects 
with parsing them. Dissecting the overall genetic risk for SCZ into biologically meaningful information related with miR-137 co-expression regulation reveals previously unreported links between miR-137 targets and in vivo phenotypes of SCZ. We suggest that the functional role of miR137 in emotion processing is mediated by the co-expression of a gene set involved in SCZ risk.

Common variation in target genes of miR-137 is related with emotion processing.

We show that miR-137 is associated with co-expression of SCZ risk genes in human nonpsychiatric post-mortem PFC. Results are consistent across independent samples, thus reducing the chance of type I error $(3,36)$. In silico predictions of miR-137 association with Darkorange coexpression are supported by in vitro experiments. Prior evidence that miR-137 SCZ-associated variants are related with working memory and not emotion processing brain activity is replicated, and we identify a role of miR-137 in emotion processing. Importantly, the sizes of the fMRI cohorts previously tested $(25,28)$ ranged up to 86 participants, whereas here we studied 358 individuals overall. As we assessed healthy individuals without familial psychiatric history, our findings speak to the role of physiological variation in a subset of miR-137 targets. In particular, greater miR-137 target gene expression (mostly associated with lower miR-137 expression in the Brainspan dataset and in our experimental validation - Figure S6) - in turn linked with greater SCZ risk (21) - is associated with greater prefrontal activity and lower prefrontal-amygdala coupling during emotion processing. These findings are consistent with prior evidence of greater prefrontal activity in individuals carrying rs1625579 risk allele (23) and with decreased prefrontal-amygdala connectivity associated with SCZ risk (37). Notably, our findings link lateral prefrontal cortex with amygdala, rather than ventromedial prefrontal regions investigated in previous studies (25), like in previous reports associating disrupted lateral prefrontal-amygdala connectivity with genetic risk for SCZ (38). We propose that lower miR-137 expression in the prefrontal cortex is associated with risk for SCZ 
by an increase of target gene expression. Some of the SCZ risk genes are co-expressed in the pathway we identified here, which is reproducibly related with increased prefrontal activity during emotion processing and decreased prefrontal-amygdala coupling.

Co-expression of miR-137 target genes.

The over-representation of synaptic and neurodevelopmental genes in Darkorange is consistent with prior evidence about miR-137 functions $(39,40)$. For example, He and coworkers (41) found that miR-137 expression was associated with synaptic protein levels in mouse hippocampus relating such dysfunction with Synaptotagmin-1, the main calcium sensor regulating brain synaptic vesicle release (42). In mice, overexpression of the Synaptotagmin-1 coding gene Syt 1 antagonizes deficits caused by miR-137 OE (43). Therefore, it is noteworthy that Syt1 is a member of Darkorange and a DDEG.

Two further relevant genes are GRM5 and GSK3B. GRM5, coding for mGluR5, has been implicated in SCZ (44) and in affective phenotypes (45). Interestingly, mGlu5 acts via a pathway mediated by activation of phospholipase $\mathrm{C}$ and protein kinase $\mathrm{C}(46)$, a pathway also represented in Darkorange (PLCBI and $P R K C B)$. Similarly, many studies have implicated the GlycogeneSynthetase-Kinase-3beta (GSK3B) gene in emotion processing (47) and in SCZ (48). GSK3B ranks among the most connected genes within Darkorange (Table S4), making it a potential hub of coexpression. These findings are consistent with prior reports implicating $G S K 3 B$ variants in SCZ intermediate phenotypes (48-50).

\section{Limitations}

We identified Darkorange based on over-representation of predicted miR-137 targets. About 
50 targets have been experimentally validated (26), but the number is likely much larger. As parsing 50 genes across 51 co-expression modules would be inadequate for enrichment analysis, we relied on predictions, as done elsewhere (28). The reliability of these predictions limits our inferences. We endeavored to address this limitation by in vitro experiments showing that Darkorange stands out at the intersection between bioinformatics and findings in neuroblastoma cells (Figure 2). Notably, the overexpression we employed may be above the physiological range of variation, hence potentially exceeding the physiological response to miR-137. Although we do not have evidence that DDEGs are directly, rather than indirectly, targeted by miR-137, this evidence suggests a miR-137 association with Darkorange. Finally, relative to our previous work (31), we traded sample size for homogeneity, by selecting only neurotypical individuals of Caucasian ancestry in order to match the SCZ-GWAS (19) and our fMRI cohort.

\section{Conclusions}

As in prior reports, polygenic SCZ risk enriched for miR-137 target genes is associated with WM-related PFC activity. Furthermore, we identified the regulation of Darkorange gene coexpression as a mechanism of miR-137 involvement in risk for SCZ. Darkorange co-eQTLs were associated with prefrontal activity and connectivity during emotion processing, in line with previous knowledge about miR-137 and emotion processing. We propose that low miR-137 expression in the prefrontal cortex is associated with SCZ risk via the increased expression of specific risk genes which is associated with increased prefrontal activation during emotion processing.

The study of co-expression in post-mortem tissue and neuroimaging phenotypes disentangles the functional genetics of gene regulators with clinical relevance beyond PRS approaches. The biological role of miR-137 in SCZ likely acts via co-expressed gene sets. Here we have identified one of such sets related with emotion processing brain activity and connectivity in humans. 


\section{Materials and Methods}

\section{Experimental Design}

Identification of a Gene Co-expression Network in the human prefrontal cortex.

We used CommonMind Consortium RNA-sequencing data from post-mortem prefrontal cortex (9) to identify a gene co-expression network in HCs by means of Weighted Gene Coexpression Network Analysis (WGCNA)(51). A dataset comparable with the fMRI sample in terms of age and ancestry included 147 neurotypical Caucasian adults (demographics in Table S1; SI-1.1). After correcting the expression data for confounding variables, we computed WGCNA as previously reported (31) on 17,173 genes. Here we used Spearman's correlation as a weighted measure of genegene relationships (52) to limit the impact of deviations from normality in expression data (53) (SI$1.1)$.

We validated the discovered gene-gene relationships using three independent datasets (BrainEAC (54) Frontal Cortex [N=123], Braincloud PFC BA46 [N=59](13) and GTEx (55) PFC BA9, [N=84], Table S1). A permutation approach served to compare module cohesiveness (56)(SI1.1). We assessed the replication of each module in the three datasets and performed a meta-analysis to obtain module-wise replication p-values (sum-log Fisher's method; $\alpha_{\text {Bonferroni }}=.05 /\left(\mathrm{N}_{\text {modules }} \times 3\right)$ ).

\section{Module prioritization and functional analysis}

We assessed the overlap between genes in the successfully replicated co-expression modules and miR-137 target genes derived via a meta-analysis of four miRNA target databases (57-60). Then, we assessed the over-representation of genes located in loci previously associated with SCZ at genome-wide significance (19) within the miR-137-enriched modules (Table S2; SI-1.2). We characterized the modules of interest using the Gene Ontology Database (PANTHER)(61). We 
investigated module function via enrichments for chromosomal locus (62), cell-type, and brain region-specific expression pattern (63) during neurodevelopment. To further assess the potential relationship between risk and gene expression, we also tested the over-representation of transcriptome-wide association study $(\mathrm{TWAS})(11,17,64)$ variants in the selected modules.

miR-137 targets in the prioritized modules and topology of schizophrenia risk genes

As there was no direct quantification of miR-137 in the dataset we used to compute the network, we used the Brainspan dorsolateral prefrontal cortex data to verify the association between miR-137 and the modules of interest (only $\mathrm{N}=10$ had a miR-137 quantification available). We computed Pearson's correlations between miR-137 and each module gene; then, we assessed the correlation difference between miR-137 targets and non-targets to support the set of targets we used.

The fact that the prioritized modules show significant enrichment for both miR-137 and SCZ gene lists does not necessarily imply that the involved miR-137 targets are linked to SCZ genetic risk. Therefore, we overlapped the gene lists to identify miR-137 targets associated with SCZ risk within modules of interest and studied the topology of the network. Specifically, we explored whether miR137 targets were connected to SCZ risk genes above chance level and whether SCZ risk genes were more related with targets than with non-targets in the prioritized modules. To this aim, we used intramodular connectivity, a standard output of WGCNA, and assessed the difference of connectivity between targets/non-targets and SCZ risk genes (Wilcoxon rank-sum tests). To test the relationship of SCZ risk genes with targets and non-targets, we computed module-wise correlation matrices and assessed the difference in correlations with Wilcoxon rank-sum tests.

Experimental validation in neuroblastoma cells and data analysis. 
We modulated the expression of miR-137 in neuroblastoma Neuro2A cells to assess WGCNA-based predictions of a link between miR-137 and the expression of genes belonging to the prioritized modules (SI-1.3). A CRISPR-Cas9 approach involving a plasmid expressing both Cas9 and a specific small guide RNA was used to generate miR-137 KO cells. A PcDNA3.2/V5 mmu-mir137 plasmid expressing miR137 was used for OE conditions (65). Impact of dose-dependent linear changes in miR-137 expression on transcriptome-wide expression was evaluated using microarrays (Mouse Gene 2.0 ST microarrays, Affymetrix/Thermo-Fisher). In the linear models, gene expression was the dependent variable and miR-137 quantification was the predictor. We thresholded results ad $\mathrm{q}_{\mathrm{FDR}}<.05$ to obtain a list of dose-dependently expressed genes (DDEGs) and assessed DDEGs overrepresentation in the target modules (SI-1.3).

Biological genetic stratification of miR-137 target genes: the Polygenic Co-expression Index.

In order to test systems-level phenotypes associated with the module of interest, we generated a Polygenic Co-expression Index (PCI $\left.\mathrm{PiR}_{\mathrm{m}-137}\right)$. We identified SNPs in the module genes associated with the first principal component of module gene expression (a measure of co-expression of the whole module) and combined them into the $\mathrm{PCI}_{\mathrm{miR}-137}(13,30,66)(\mathrm{SI}-1.4)$. We estimated the effect of allelic dosage via a Robust Linear Model (rlm function - robust $\mathrm{R}$ package) and ranked SNPs according to their p-value (31). Following prior reports, we assessed the top 50 ranked SNPs for PCI computation (31), and computed the $\mathrm{PCI}_{\mathrm{miR}-137}$ picking the top set of SNPs that replicated the association with co-expression in the largest replication dataset we had available (BrainEAC; SI-1.4).

Neuroimaging study.

Participants. We recruited 358 healthy volunteers distributed in a discovery cohort of 222 participants who had both EP and WM data available and a replication cohort of 136 participants for 
the emotion processing task (Table S1). We evaluated a single working memory cohort because the

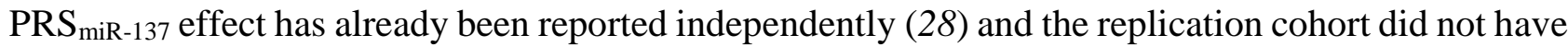
working memory scans. Inclusion/exclusion criteria have been described elsewhere (SI-1.5)(67). Participants signed an informed consent complying with the Declaration of Helsinki after full explanation of all procedures approved by the local ethics committee.

Genetic score computation. We genotyped all participants (68) and performed imputation using Sanger Imputation Service and 1000 Genomes Project Phase 3 reference panel (including prephasing and imputation with SHAPEIT2+PBWT $(69,70)$; genomic coordinates on GRCh37/hg19 genomic reference build). We filtered out SNPs with missing rate >.05, Hardy-Weinberg equilibrium $\mathrm{p}<10^{-6}$, and minor allele frequency (MAF) $<.01$ and computed genomic eigenvariates to control for population stratification (71).

The PCI $\mathrm{miR}_{137}$ is the average of the co-expression effects of the top-ranked SNP alleles selected in the post-mortem study, which replicated in BrainEAC. Instead, we computed the PRS $S_{\text {miR- }}$ 137 for SCZ using standard procedures (71) with the miR-137 target gene list and SNPs reported in the original article (28)(SNP-level significance thresholds: $\left.\mathrm{p}<10^{-5}, \mathrm{p}<.05, \mathrm{p}<.5\right)$. SI-1.6 reports further scores computed to control for possible confounders and the pertinent results.

Neuropsychological tasks. Our emotion processing Faces task (72-74) presented participants with angry, fearful, happy and neutral facial expressions from a validated set of facial pictures (SI-1.5). The N-back task probes working memory and has been widely used in neuroimaging (75-77). Participants performed three runs of a block design version of the task: 1-Back vs. 0-Back; 2-Back vs. 0-Back, and 3-Back vs. 0-Back, each lasting $240 \mathrm{s.}$

\section{Statistical Analysis}

fMRI data collection, pre-processing, and analysis followed standard procedures (SI-1.5)(13). We used SPM12 to perform multiple regression analyses for both tasks and for the two samples 
separately. For the co-expression analysis, we used the linear and quadratic terms of $\mathrm{PCI}_{\mathrm{miR}-137}(30$, 66, 78) as predictors and age, gender, and five genomic eigenvariates as covariates. For the PRS $\mathrm{miR}_{\text {- }}$ 137 analyses, we used PRSs as predictors and the same covariates reported above. In the working memory/emotion processing sample, we report results surviving $\mathrm{p}_{\mathrm{FWE}}<.05$ threshold at whole brain level masked by task activity. In the emotion processing replication sample, we used the cluster detected in the discovery analysis and performed a small volume correction $\left(\mathrm{p}_{\mathrm{SVC}}<.05\right)$.

To link miR-137 target gene predicted expression in the prefrontal cortex with prefrontalamygdala functional coupling, we additionally performed a connectivity analysis using a geneticphysiological interaction approach (79). Specifically, we used the prefrontal seed associated with the PCI $\mathrm{I}_{\mathrm{miR}-137}$ and extracted movement- and task-corrected estimates from the bilateral amygdala (WFU Pickatlas (25); SI 1.5). 
Acknowledgments: CommonMind Consortium data were generously provided to GP by the NIMH and CommonMind Consortium. Data acquisition was possible thanks to the work by Dr. Linda A. Antonucci, Dr. Barbara Gelao, Dr. Marina Mancini, Dr. Annamaria Porcelli, and Dr. Paolo Taurisano. We gratefully acknowledge the work by Prof. Roberto Bellotti and Dr. Sabina Tangaro (Department of Physics - University of Bari Aldo Moro), Dr. Lucia Colagiorgio, Dr. Anna Monda, Annalisa Lella and Elisabetta Volpe (Department of Basic Medical Science, Neuroscience, and Sense Organs - University of Bari Aldo Moro), who contributed to data analysis.

Funding: This project has received funding from the European Union Seventh Framework Programme for research, technological development and demonstration under grant agreement no. 602450 (IMAGEMEND). This work was also supported by a Canadian Institutes of Medical Research (CIHR) MOP-136916 to Jean-Martin Beaulieu; a "Capitale Umano ad Alta Qualificazione" grant by Fondazione Con Il Sud and by a Collaboration Grant from Itel srl, awarded to Alessandro Bertolino; by the Residency School in Psychiatry of the University of Bari Aldo Moro; by the Consejo Nacional de Investigaciones Científicas y Técnicas (CONICET), Argentina (to Mariana Nair Castro); and by Hoffmann-La Roche through a Collaboration Grant awarded to Giulio Pergola and through financial support to Enrico Domenici and Juergen Dukart by Hoffmann-La Roche Ltd. Giulio Pergola's position is currently funded by the European Union's Horizon 2020 research and innovation program under the Marie Sklodowska-Curie grant agreement no. 798181 (FLOURISH). Jean-Martin Beaulieu in Canada Research Chair (Tier1) in Molecular Psychiatry. This paper reflects only the author's views and the European Union and Research Executive Agency are not liable for any use that may be made of the information contained therein. 
Competing interests: Alessandro Bertolino received consulting fees by Biogen and lecture fees by Otsuka, Janssen, Lundbeck. Giuseppe Blasi received lecture fees by Lundbeck. Antonio Rampino received travel fees by Lundbeck. Marco Papalino received travel fees by Newron Pharmaceuticals. Enrico Domenici was an employee of Hoffmann-La Roche Ltd. (2010-2015) and has received research support from Hoffmann-La Roche Ltd. in the period 2016-2018. All other authors have no biomedical financial interests and no potential conflicts of interest. 
Table 1. PGC loci and genes overlapping with the module Darkorange and miR-137 targets.

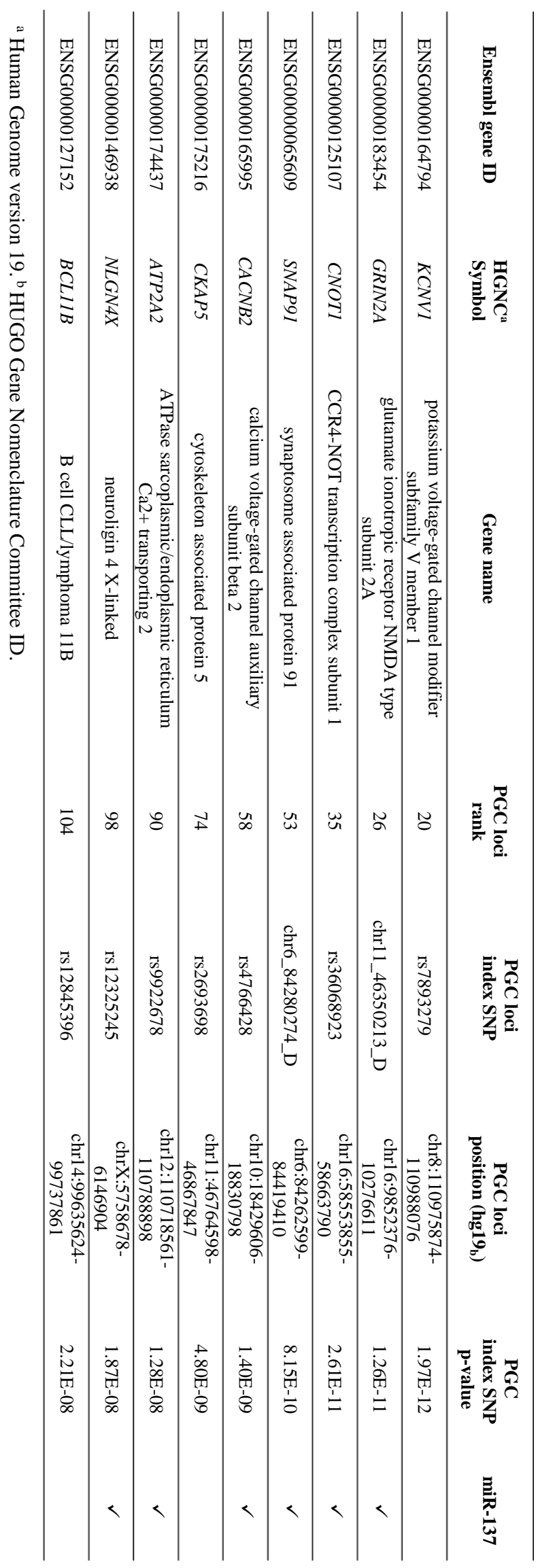


Table 2. SNPs associated with the module Darkorange.

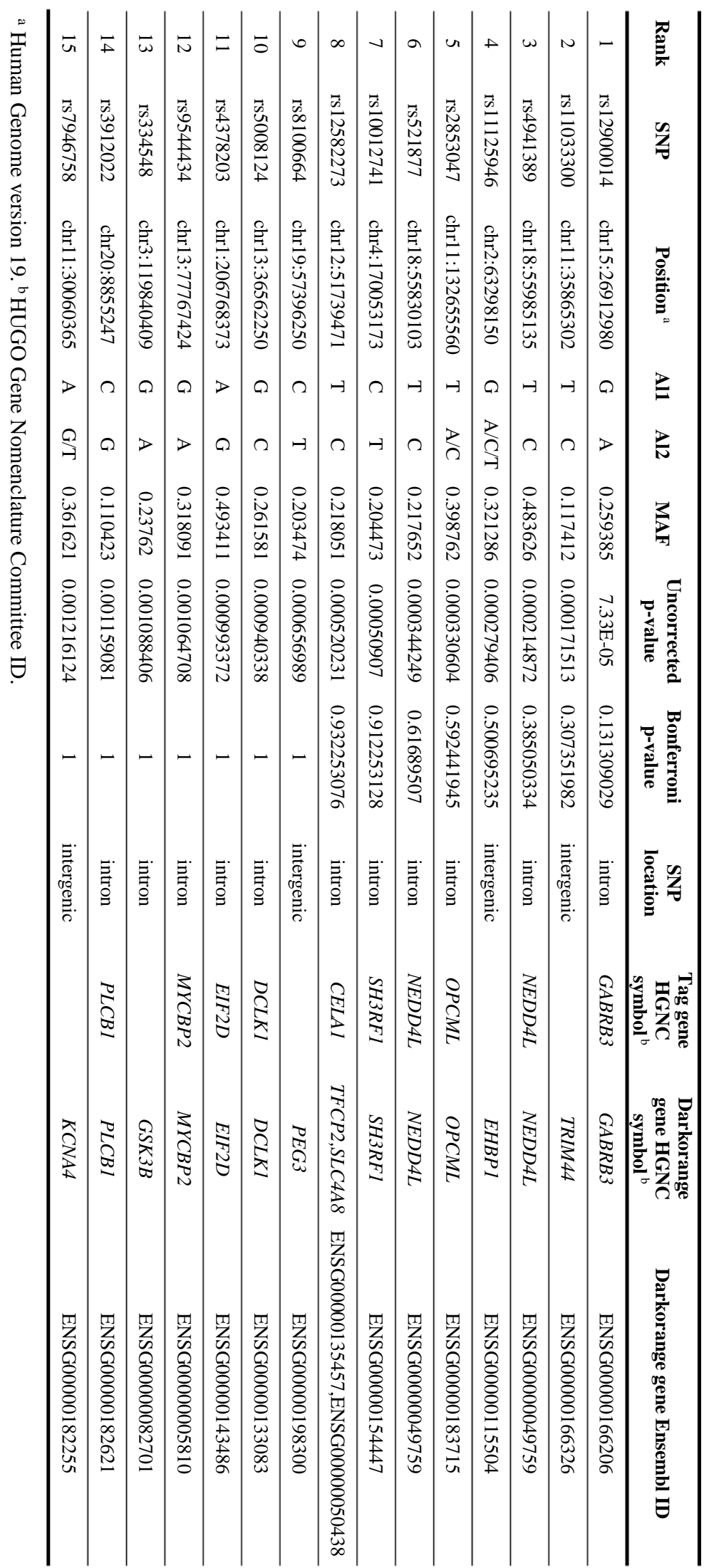




\section{Figure captions:}

\section{Figure 1. Study design.}

Figure 2. Module prioritization. Features of the Darkorange module. A. Intersection between Darkorange genes, Psychiatric Genomic Consortium (PGC) SCZ genes, and miR-137 targetome bioinformatic predictions in humans. B. Gene ontologies associated with Darkorange genes. Reported p-values are corrected for multiple comparisons. C. Overview of the over-representation in all replicated modules (28 out of 51) of miR-137 predicted targets in humans, of SCZ genes, of genetic variants associated with SCZ (MAGMA), and of dose-dependent expressed genes in neuroblastoma cells (DDEGs). The colormap highlights the specificity of Darkorange relative to other modules. D. Scatter plot illustrates the enrichment of each replicated module for miR-137 targets as assessed in neuroblastoma cells and in bioinformatic predictions in humans. The two assessments co-vary except for outliers Blue and Red.

Figure 3. fMRI results. Top panels: render of the brain activation associated with $\mathrm{PCI}_{\mathrm{miR}-137}$ during EP in the discovery (A.) and replication sample (B.). C. Render of the brain activation associated

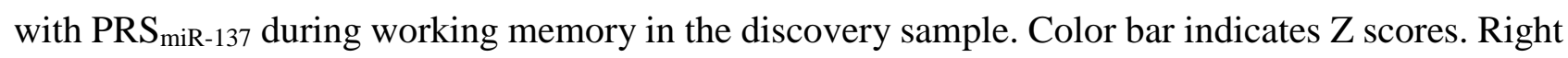
in the figures is right in the brain. Each scatterplot refers to the cluster highlighted in the render on its top. In the scatterplots, axes are scaled with mean $=0$ and standard deviation $=1$. 


\section{References}

1. P. F. Sullivan, K. S. Kendler, M. C. Neale, Schizophrenia as a complex trait: evidence from a meta-analysis of twin studies. Archives of general psychiatry 60, 1187-1192 (2003).

2. P. McGuffin, B. Riley, R. Plomin, Genomics and behavior. Toward behavioral genomics. Science 291, 1232-1249 (2001).

3. N. N. Parikshak, M. J. Gandal, D. H. Geschwind, Systems biology and gene networks in neurodevelopmental and neurodegenerative disorders. Nat Rev Genet 16, 441-458 (2015).

4. K. Kauppi, L. T. Westlye, M. Tesli, F. Bettella, C. L. Brandt, M. Mattingsdal, T. Ueland, T. Espeseth, I. Agartz, I. Melle, S. Djurovic, O. A. Andreassen, Polygenic risk for schizophrenia associated with working memory-related prefrontal brain activation in patients with schizophrenia and healthy controls. Schizophrenia bulletin 41, 736-743 (2015).

5. A. Krug, B. Dietsche, R. Zollner, D. Yuksel, M. M. Nothen, A. J. Forstner, M. Rietschel, U. Dannlowski, B. T. Baune, R. Maier, S. H. Witt, T. Kircher, Polygenic risk for schizophrenia affects working memory and its neural correlates in healthy subjects. Schizophrenia research, (2018).

6. J. A. Miller, M. A. Scult, E. D. Conley, Q. Chen, D. R. Weinberger, A. R. Hariri, Effects of Schizophrenia Polygenic Risk Scores on Brain Activity and Performance During Working Memory Subprocesses in Healthy Young Adults. Schizophrenia bulletin 44, 844-853 (2018).

7. I. Dzafic, H. Burianova, S. Periyasamy, B. Mowry, Association between schizophrenia polygenic risk and neural correlates of emotion perception. Psychiatry research. Neuroimaging 276, 33-40 (2018).

8. S. Erk, S. Mohnke, S. Ripke, T. A. Lett, I. M. Veer, C. Wackerhagen, O. Grimm, N. Romanczuk-Seiferth, F. Degenhardt, H. Tost, M. Mattheisen, T. W. Muhleisen, K. Charlet, N. Skarabis, F. Kiefer, S. Cichon, S. H. Witt, M. M. Nothen, M. Rietschel, A. Heinz, A. Meyer-Lindenberg, H. Walter, Functional neuroimaging effects of recently discovered genetic risk loci for schizophrenia and polygenic risk profile in five RDoC subdomains. Translational psychiatry 7, e997 (2017).

9. M. Fromer, P. Roussos, S. K. Sieberts, J. S. Johnson, D. H. Kavanagh, T. M. Perumal, D. M. Ruderfer, E. C. Oh, A. Topol, H. R. Shah, L. L. Klei, R. Kramer, D. Pinto, Z. H. Gumus, A. E. Cicek, K. K. Dang, A. Browne, C. Lu, L. Xie, B. Readhead, E. A. Stahl, J. Xiao, M. Parvizi, T. Hamamsy, J. F. Fullard, Y. C. Wang, M. C. Mahajan, J. M. Derry, J. T. Dudley, S. E. Hemby, B. A. Logsdon, K. Talbot, T. Raj, D. A. Bennett, P. L. De Jager, J. Zhu, B. Zhang, P. F. Sullivan, A. Chess, S. M. Purcell, L. A. Shinobu, L. M. Mangravite, H. Toyoshiba, R. E. Gur, C. G. Hahn, D. A. Lewis, V. Haroutunian, M. A. Peters, B. K. Lipska, J. D. Buxbaum, E. E. Schadt, K. Hirai, K. Roeder, K. J. Brennand, N. Katsanis, E. Domenici, B. Devlin, P. Sklar, Gene expression elucidates functional impact of polygenic risk for schizophrenia. Nature neuroscience 19, 1442-1453 (2016).

10. A. E. Jaffe, R. E. Straub, J. H. Shin, R. Tao, Y. Gao, L. Collado-Torres, T. Kam-Thong, H. S. Xi, J. Quan, Q. Chen, C. Colantuoni, W. S. Ulrich, B. J. Maher, A. Deep-Soboslay, C. BrainSeq, A. J. Cross, N. J. Brandon, J. T. Leek, T. M. Hyde, J. E. Kleinman, D. R. Weinberger, Developmental and genetic regulation of the human cortex transcriptome illuminate schizophrenia pathogenesis. Nature neuroscience 21, 1117-1125 (2018).

11. A. Gusev, N. Mancuso, H. Won, M. Kousi, H. K. Finucane, Y. Reshef, L. Song, A. Safi, C. Schizophrenia Working Group of the Psychiatric Genomics, S. McCarroll, B. M. Neale, R. A. Ophoff, M. C. O'Donovan, G. E. Crawford, D. H. Geschwind, N. Katsanis, P. F. Sullivan, B. Pasaniuc, A. L. Price, Transcriptome-wide association study of schizophrenia and chromatin activity yields mechanistic disease insights. Nature genetics 50, 538-548 (2018). 
12. E. Radulescu, A. E. Jaffe, R. E. Straub, Q. Chen, J. H. Shin, T. M. Hyde, J. E. Kleinman, D. R. Weinberger, Identification and prioritization of gene sets associated with schizophrenia risk by co-expression network analysis in human brain. bioRxiv, (2018).

13. G. Pergola, P. Di Carlo, E. D'Ambrosio, B. Gelao, L. Fazio, M. Papalino, A. Monda, G. Scozia, B. Pietrangelo, M. Attrotto, J. A. Apud, Q. Chen, V. S. Mattay, A. Rampino, G. Caforio, D. R. Weinberger, G. Blasi, A. Bertolino, DRD2 co-expression network and a related polygenic index predict imaging, behavioral and clinical phenotypes linked to schizophrenia. Translational psychiatry 7, e1006 (2017).

14. N. N. Parikshak, V. Swarup, T. G. Belgard, M. Irimia, G. Ramaswami, M. J. Gandal, C. Hartl, V. Leppa, L. T. Ubieta, J. Huang, J. K. Lowe, B. J. Blencowe, S. Horvath, D. H. Geschwind, Genome-wide changes in lncRNA, splicing, and regional gene expression patterns in autism. Nature 540, 423-427 (2016).

15. W. Sun, J. Poschmann, R. Cruz-Herrera Del Rosario, N. N. Parikshak, H. S. Hajan, V. Kumar, R. Ramasamy, T. G. Belgard, B. Elanggovan, C. C. Wong, J. Mill, D. H. Geschwind, S. Prabhakar, Histone Acetylome-wide Association Study of Autism Spectrum Disorder. Cell 167, 1385-1397 e1311 (2016).

16. X. Liu, Y. I. Li, J. K. Pritchard, Trans Effects on Gene Expression Can Drive Omnigenic Inheritance. Cell 177, 1022-1034 e1026 (2019).

17. M. J. Gandal, P. Zhang, E. Hadjimichael, R. L. Walker, C. Chen, S. Liu, H. Won, H. van Bakel, M. Varghese, Y. Wang, A. W. Shieh, J. Haney, S. Parhami, J. Belmont, M. Kim, P. Moran Losada, Z. Khan, J. Mleczko, Y. Xia, R. Dai, D. Wang, Y. T. Yang, M. Xu, K. Fish, P. R. Hof, J. Warrell, D. Fitzgerald, K. White, A. E. Jaffe, E. C. Psych, M. A. Peters, M. Gerstein, C. Liu, L. M. Iakoucheva, D. Pinto, D. H. Geschwind, Transcriptome-wide isoformlevel dysregulation in ASD, schizophrenia, and bipolar disorder. Science 362, (2018).

18. M. E. Hauberg, P. Roussos, J. Grove, A. D. Borglum, M. Mattheisen, C. Schizophrenia Working Group of the Psychiatric Genomics, Analyzing the Role of MicroRNAs in Schizophrenia in the Context of Common Genetic Risk Variants. JAMA psychiatry 73, 369377 (2016).

19. C. Schizophrenia Working Group of the Psychiatric Genomics, Biological insights from 108 schizophrenia-associated genetic loci. Nature 511, 421-427 (2014).

20. K. Sakamoto, J. J. Crowley, A comprehensive review of the genetic and biological evidence supports a role for MicroRNA-137 in the etiology of schizophrenia. American journal of medical genetics. Part B, Neuropsychiatric genetics : the official publication of the International Society of Psychiatric Genetics 177, 242-256 (2018).

21. I. Guella, A. Sequeira, B. Rollins, L. Morgan, F. Torri, T. G. van Erp, R. M. Myers, J. D. Barchas, A. F. Schatzberg, S. J. Watson, H. Akil, W. E. Bunney, S. G. Potkin, F. Macciardi, M. P. Vawter, Analysis of miR-137 expression and rs1625579 in dorsolateral prefrontal cortex. Journal of psychiatric research 47, 1215-1221 (2013).

22. Z. Zhang, T. Yan, Y. Wang, Q. Zhang, W. Zhao, X. Chen, J. Zhai, M. Chen, B. Du, X. Deng, F. Ji, Y. Xiang, H. Wu, J. Song, Q. Dong, C. Chen, J. Li, Polymorphism in schizophrenia risk gene MIR137 is associated with the posterior cingulate Cortex's activation and functional and structural connectivity in healthy controls. NeuroImage. Clinical 19, 160-166 (2018).

23. T. G. van Erp, I. Guella, M. P. Vawter, J. Turner, G. G. Brown, G. McCarthy, D. N. Greve, G. H. Glover, V. D. Calhoun, K. O. Lim, J. R. Bustillo, A. Belger, J. M. Ford, D. H. Mathalon, M. Diaz, A. Preda, D. Nguyen, F. Macciardi, S. G. Potkin, Schizophrenia miR-137 locus risk genotype is associated with dorsolateral prefrontal cortex hyperactivation. Biological psychiatry 75, 398-405 (2014).

24. H. C. Whalley, M. Papmeyer, L. Romaniuk, E. Sprooten, E. C. Johnstone, J. Hall, S. M. Lawrie, K. L. Evans, H. P. Blumberg, J. E. Sussmann, A. M. McIntosh, Impact of a microRNA MIR137 susceptibility variant on brain function in people at high genetic risk of schizophrenia 
or bipolar disorder. Neuropsychopharmacology : official publication of the American College of Neuropsychopharmacology 37, 2720-2729 (2012).

25. O. Mothersill, D. W. Morris, S. Kelly, E. J. Rose, C. Fahey, C. O'Brien, R. Lyne, R. Reilly, M. Gill, A. P. Corvin, G. Donohoe, Effects of MIR137 on fronto-amygdala functional connectivity. NeuroImage 90, 189-195 (2014).

26. E. Mahmoudi, M. J. Cairns, MiR-137: an important player in neural development and neoplastic transformation. Molecular psychiatry 22, 44-55 (2017).

27. S. G. Potkin, F. Macciardi, G. Guffanti, J. H. Fallon, Q. Wang, J. A. Turner, A. Lakatos, M. F. Miles, A. Lander, M. P. Vawter, X. Xie, Identifying gene regulatory networks in schizophrenia. NeuroImage 53, 839-847 (2010).

28. D. Cosgrove, D. Harold, O. Mothersill, R. Anney, M. J. Hill, N. J. Bray, G. Blokland, T. Petryshen, C. Wellcome Trust Case Control, A. Richards, K. Mantripragada, M. Owen, M. C. O'Donovan, M. Gill, A. Corvin, D. W. Morris, G. Donohoe, MiR-137-derived polygenic risk: effects on cognitive performance in patients with schizophrenia and controls. Translational psychiatry 7, e1012 (2017).

29. I. Esterlis, S. E. Holmes, P. Sharma, J. H. Krystal, C. DeLorenzo, Metabotropic Glutamatergic Receptor 5 and Stress Disorders: Knowledge Gained From Receptor Imaging Studies. Biological psychiatry, (2017).

30. L. Fazio, G. Pergola, M. Papalino, P. Di Carlo, A. Monda, B. Gelao, N. Amoroso, S. Tangaro, A. Rampino, T. Popolizio, A. Bertolino, G. Blasi, Transcriptomic context of DRD1 is associated with prefrontal activity and behavior during working memory. Proceedings of the National Academy of Sciences of the United States of America, (2018).

31. G. Pergola, P. Di Carlo, A. E. Jaffe, M. Papalino, Q. Chen, T. M. Hyde, J. E. Kleinman, J. H. Shin, A. Rampino, G. Blasi, D. R. Weinberger, A. Bertolino, Prefrontal Coexpression of Schizophrenia Risk Genes Is Associated With Treatment Response in Patients. Biological psychiatry 86, 45-55 (2019).

32. L. A. Antonucci, P. Di Carlo, R. Passiatore, M. Papalino, A. Monda, N. Amoroso, S. Tangaro, P. Taurisano, A. Rampino, F. Sambataro, T. Popolizio, A. Bertolino, G. Pergola, G. Blasi, Thalamic connectivity measured with fMRI is associated with a polygenic index predicting thalamo-prefrontal gene co-expression. Brain structure \& function 224, 1331-1344 (2019).

33. C. A. de Leeuw, J. M. Mooij, T. Heskes, D. Posthuma, MAGMA: generalized gene-set analysis of GWAS data. PLoS computational biology 11, e1004219 (2015).

34. N. G. Skene, J. Bryois, T. E. Bakken, G. Breen, J. J. Crowley, H. A. Gaspar, P. GiustiRodriguez, R. D. Hodge, J. A. Miller, A. B. Munoz-Manchado, M. C. O'Donovan, M. J. Owen, A. F. Pardinas, J. Ryge, J. T. R. Walters, S. Linnarsson, E. S. Lein, C. Major Depressive Disorder Working Group of the Psychiatric Genomics, P. F. Sullivan, J. Hjerling-Leffler, Genetic identification of brain cell types underlying schizophrenia. Nature genetics 50, 825833 (2018).

35. K. Ohi, T. Shimada, Y. Nitta, H. Kihara, H. Okubo, T. Uehara, Y. Kawasaki, Specific gene expression patterns of 108 schizophrenia-associated loci in cortex. Schizophrenia research 174, 35-38 (2016).

36. R. Whelan, H. Garavan, When optimism hurts: inflated predictions in psychiatric neuroimaging. Biological psychiatry 75, 746-748 (2014).

37. H. Cao, A. Bertolino, H. Walter, M. Schneider, A. Schafer, P. Taurisano, G. Blasi, L. Haddad, O. Grimm, K. Otto, L. Dixson, S. Erk, S. Mohnke, A. Heinz, N. Romanczuk-Seiferth, T. W. Muhleisen, M. Mattheisen, S. H. Witt, S. Cichon, M. Noethen, M. Rietschel, H. Tost, A. Meyer-Lindenberg, Altered Functional Subnetwork During Emotional Face Processing: A Potential Intermediate Phenotype for Schizophrenia. JAMA psychiatry 73, 598-605 (2016).

38. T. Quarto, I. Paparella, D. De Tullio, G. Viscanti, L. Fazio, P. Taurisano, R. Romano, A. Rampino, R. Masellis, T. Popolizio, P. Selvaggi, G. Pergola, A. Bertolino, G. Blasi, Familial Risk and a Genome-Wide Supported DRD2 Variant for Schizophrenia Predict Lateral 
Prefrontal-Amygdala Effective Connectivity During Emotion Processing. Schizophrenia bulletin 44, 834-843 (2018).

39. R. D. Smrt, K. E. Szulwach, R. L. Pfeiffer, X. Li, W. Guo, M. Pathania, Z. Q. Teng, Y. Luo, J. Peng, A. Bordey, P. Jin, X. Zhao, MicroRNA miR-137 regulates neuronal maturation by targeting ubiquitin ligase mind bomb-1. Stem cells 28, 1060-1070 (2010).

40. K. E. Szulwach, X. Li, R. D. Smrt, Y. Li, Y. Luo, L. Lin, N. J. Santistevan, W. Li, X. Zhao, P. Jin, Cross talk between microRNA and epigenetic regulation in adult neurogenesis. The Journal of cell biology 189, 127-141 (2010).

41. E. He, M. A. G. Lozano, S. Stringer, K. Watanabe, K. Sakamoto, F. den Oudsten, F. Koopmans, S. N. Giamberardino, A. Hammerschlag, L. N. Cornelisse, K. W. Li, J. van Weering, D. Posthuma, A. B. Smit, P. F. Sullivan, M. Verhage, MIR137 schizophreniaassociated locus controls synaptic function by regulating synaptogenesis, synapse maturation and synaptic transmission. Human molecular genetics 27, 1879-1891 (2018).

42. C. N. Egbujo, D. Sinclair, C. G. Hahn, Dysregulations of Synaptic Vesicle Trafficking in Schizophrenia. Current psychiatry reports 18, 77 (2016).

43. S. Siegert, J. Seo, E. J. Kwon, A. Rudenko, S. Cho, W. Wang, Z. Flood, A. J. Martorell, M. Ericsson, A. E. Mungenast, L. H. Tsai, The schizophrenia risk gene product miR-137 alters presynaptic plasticity. Nature neuroscience 18, 1008-1016 (2015).

44. R. S. Devon, S. Anderson, P. W. Teague, W. J. Muir, V. Murray, A. J. Pelosi, D. H. Blackwood, D. J. Porteous, The genomic organisation of the metabotropic glutamate receptor subtype 5 gene, and its association with schizophrenia. Molecular psychiatry 6, 311-314 (2001).

45. S. Terbeck, F. Akkus, L. P. Chesterman, G. Hasler, The role of metabotropic glutamate receptor 5 in the pathogenesis of mood disorders and addiction: combining preclinical evidence with human Positron Emission Tomography (PET) studies. Frontiers in neuroscience 9, 86 (2015).

46. M. F. Olive, A. J. McGeehan, J. R. Kinder, T. McMahon, C. W. Hodge, P. H. Janak, R. O. Messing, The mGluR5 antagonist 6-methyl-2-(phenylethynyl)pyridine decreases ethanol consumption via a protein kinase $\mathrm{C}$ epsilon-dependent mechanism. Molecular pharmacology 67, 349-355 (2005).

47. T. Del'Guidice, C. Latapy, A. Rampino, J. Khlghatyan, M. Lemasson, B. Gelao, T. Quarto, G. Rizzo, A. Barbeau, C. Lamarre, A. Bertolino, G. Blasi, J. M. Beaulieu, FXR1P is a GSK3beta substrate regulating mood and emotion processing. Proceedings of the National Academy of Sciences of the United States of America 112, E4610-4619 (2015).

48. A. Rampino, A. Marakhovskaia, T. Soares-Silva, S. Torretta, F. Veneziani, J. M. Beaulieu, Antipsychotic Drug Responsiveness and Dopamine Receptor Signaling; Old Players and New Prospects. Frontiers in psychiatry 9, 702 (2018).

49. G. Blasi, F. Napolitano, G. Ursini, A. Di Giorgio, G. Caforio, P. Taurisano, L. Fazio, B. Gelao, M. T. Attrotto, L. Colagiorgio, G. Todarello, F. Piva, A. Papazacharias, R. Masellis, M. Mancini, A. Porcelli, R. Romano, A. Rampino, T. Quarto, M. Giulietti, B. K. Lipska, J. E. Kleinman, T. Popolizio, D. R. Weinberger, A. Usiello, A. Bertolino, Association of GSK3 beta genetic variation with GSK-3beta expression, prefrontal cortical thickness, prefrontal physiology, and schizophrenia. The American journal of psychiatry 170, 868-876 (2013).

50. E. S. Emamian, D. Hall, M. J. Birnbaum, M. Karayiorgou, J. A. Gogos, Convergent evidence for impaired AKT1-GSK3beta signaling in schizophrenia. Nature genetics 36, 131-137 (2004).

51. B. Zhang, S. Horvath, A general framework for weighted gene co-expression network analysis. Statistical applications in genetics and molecular biology 4, Article17 (2005).

52. J. Richiardi, A. Altmann, A. C. Milazzo, C. Chang, M. M. Chakravarty, T. Banaschewski, G. J. Barker, A. L. Bokde, U. Bromberg, C. Buchel, P. Conrod, M. Fauth-Buhler, H. Flor, V. Frouin, J. Gallinat, H. Garavan, P. Gowland, A. Heinz, H. Lemaitre, K. F. Mann, J. L. 
Martinot, F. Nees, T. Paus, Z. Pausova, M. Rietschel, T. W. Robbins, M. N. Smolka, R. Spanagel, A. Strohle, G. Schumann, M. Hawrylycz, J. B. Poline, M. D. Greicius, I. consortium, BRAIN NETWORKS. Correlated gene expression supports synchronous activity in brain networks. Science 348, 1241-1244 (2015).

53. S. Kumari, J. Nie, H. S. Chen, H. Ma, R. Stewart, X. Li, M. Z. Lu, W. M. Taylor, H. Wei, Evaluation of gene association methods for coexpression network construction and biological knowledge discovery. PloS one 7, e50411 (2012).

54. D. Trabzuni, M. Ryten, R. Walker, C. Smith, S. Imran, A. Ramasamy, M. E. Weale, J. Hardy, Quality control parameters on a large dataset of regionally dissected human control brains for whole genome expression studies. Journal of neurochemistry 119, 275-282 (2011).

55. G. T. Consortium, Human genomics. The Genotype-Tissue Expression (GTEx) pilot analysis: multitissue gene regulation in humans. Science 348, 648-660 (2015).

56. M. R. Johnson, K. Shkura, S. R. Langley, A. Delahaye-Duriez, P. Srivastava, W. D. Hill, O. J. Rackham, G. Davies, S. E. Harris, A. Moreno-Moral, M. Rotival, D. Speed, S. Petrovski, A. Katz, C. Hayward, D. J. Porteous, B. H. Smith, S. Padmanabhan, L. J. Hocking, J. M. Starr, D. C. Liewald, A. Visconti, M. Falchi, L. Bottolo, T. Rossetti, B. Danis, M. Mazzuferi, P. Foerch, A. Grote, C. Helmstaedter, A. J. Becker, R. M. Kaminski, I. J. Deary, E. Petretto, Systems genetics identifies a convergent gene network for cognition and neurodevelopmental disease. Nature neuroscience 19, 223-232 (2016).

57. V. Agarwal, G. W. Bell, J. W. Nam, D. P. Bartel, Predicting effective microRNA target sites in mammalian mRNAs. eLife 4, (2015).

58. N. Wong, X. Wang, miRDB: an online resource for microRNA target prediction and functional annotations. Nucleic acids research 43, D146-152 (2015).

59. S. Bandyopadhyay, R. Mitra, TargetMiner: microRNA target prediction with systematic identification of tissue-specific negative examples. Bioinformatics 25, 2625-2631 (2009).

60. I. S. Vlachos, M. D. Paraskevopoulou, D. Karagkouni, G. Georgakilas, T. Vergoulis, I. Kanellos, I. L. Anastasopoulos, S. Maniou, K. Karathanou, D. Kalfakakou, A. Fevgas, T. Dalamagas, A. G. Hatzigeorgiou, DIANA-TarBase v7.0: indexing more than half a million experimentally supported miRNA:mRNA interactions. Nucleic acids research 43, D153-159 (2015).

61. P. D. Thomas, M. J. Campbell, A. Kejariwal, H. Mi, B. Karlak, R. Daverman, K. Diemer, A. Muruganujan, A. Narechania, PANTHER: a library of protein families and subfamilies indexed by function. Genome research 13, 2129-2141 (2003).

62. J. Chen, E. E. Bardes, B. J. Aronow, A. G. Jegga, ToppGene Suite for gene list enrichment analysis and candidate gene prioritization. Nucleic acids research 37, W305-311 (2009).

63. X. Xu, A. B. Wells, D. R. O'Brien, A. Nehorai, J. D. Dougherty, Cell type-specific expression analysis to identify putative cellular mechanisms for neurogenetic disorders. The Journal of neuroscience : the official journal of the Society for Neuroscience 34, 1420-1431 (2014).

64. L. M. Huckins, A. Dobbyn, D. M. Ruderfer, G. Hoffman, W. Wang, A. F. Pardinas, V. M. Rajagopal, T. D. Als, T. N. H, K. Girdhar, J. Boocock, P. Roussos, M. Fromer, R. Kramer, E. Domenici, E. R. Gamazon, S. Purcell, C. CommonMind, C. Schizophrenia Working Group of the Psychiatric Genomics, P.-G. S. W. G. i, D. Demontis, A. D. Borglum, J. T. R. Walters, M. C. O'Donovan, P. Sullivan, M. J. Owen, B. Devlin, S. K. Sieberts, N. J. Cox, H. K. Im, P. Sklar, E. A. Stahl, Gene expression imputation across multiple brain regions provides insights into schizophrenia risk. Nature genetics 51, 659-674 (2019).

65. H. R. Chiang, L. W. Schoenfeld, J. G. Ruby, V. C. Auyeung, N. Spies, D. Baek, W. K. Johnston, C. Russ, S. Luo, J. E. Babiarz, R. Blelloch, G. P. Schroth, C. Nusbaum, D. P. Bartel, Mammalian microRNAs: experimental evaluation of novel and previously annotated genes. Genes \& development 24, 992-1009 (2010).

66. G. Pergola, P. Di Carlo, I. Andriola, B. Gelao, S. Torretta, M. T. Attrotto, L. Fazio, A. Raio, D. Albergo, R. Masellis, A. Rampino, G. Blasi, A. Bertolino, Combined effect of genetic 
variants in the GluN2B coding gene (GRIN2B) on prefrontal function during working memory performance. Psychological medicine 46, 1135-1150 (2016).

67. A. Rampino, R. M. Walker, H. S. Torrance, S. M. Anderson, L. Fazio, A. Di Giorgio, P. Taurisano, B. Gelao, R. Romano, R. Masellis, G. Ursini, G. Caforio, G. Blasi, J. K. Millar, D. J. Porteous, P. A. Thomson, A. Bertolino, K. L. Evans, Expression of DISC1-interactome members correlates with cognitive phenotypes related to schizophrenia. PloS one 9, e99892 (2014).

68. A. Rampino, P. Taurisano, G. Fanelli, M. Attrotto, S. Torretta, L. A. Antonucci, G. Miccolis, G. Pergola, G. Ursini, G. Maddalena, R. Romano, R. Masellis, P. Di Carlo, P. Pignataro, G. Blasi, A. Bertolino, A Polygenic Risk Score of glutamatergic SNPs associated with schizophrenia predicts attentional behavior and related brain activity in healthy humans.

European neuropsychopharmacology : the journal of the European College of Neuropsychopharmacology 27, 928-939 (2017).

69. O. Delaneau, J. Marchini, J. F. Zagury, A linear complexity phasing method for thousands of genomes. Nat Methods 9, 179-181 (2011).

70. R. Durbin, Efficient haplotype matching and storage using the positional Burrows-Wheeler transform (PBWT). Bioinformatics 30, 1266-1272 (2014).

71. Q. Chen, G. Ursini, A. L. Romer, A. R. Knodt, K. Mezeivtch, E. Xiao, G. Pergola, G. Blasi, R. E. Straub, J. H. Callicott, K. F. Berman, A. R. Hariri, A. Bertolino, V. S. Mattay, D. R. Weinberger, Schizophrenia polygenic risk score predicts mnemonic hippocampal activity. Brain : a journal of neurology 141, 1218-1228 (2018).

72. T. Quarto, I. Paparella, D. De Tullio, G. Viscanti, L. Fazio, P. Taurisano, R. Romano, A. Rampino, R. Masellis, T. Popolizio, P. Selvaggi, G. Pergola, A. Bertolino, G. Blasi, Familial Risk and a Genome-Wide Supported DRD2 Variant for Schizophrenia Predict Lateral Prefrontal-Amygdala Effective Connectivity During Emotion Processing. Schizophrenia bulletin, (2017).

73. A. Rampino, S. Torretta, G. Rizzo, G. Viscanti, T. Quarto, B. Gelao, L. Fazio, M. T. Attrotto, R. Masellis, G. Pergola, A. Bertolino, G. Blasi, Emotional Stability Interacts with Cortisol Levels Before fMRI on Brain Processing of Fearful Faces. Neuroscience, (2019).

74. L. A. Antonucci, G. Pergola, R. Passiatore, P. Taurisano, T. Quarto, E. Dispoto, A. Rampino, A. Bertolino, R. Cassibba, G. Blasi, The interaction between OXTR rs2268493 and perceived maternal care is associated with amygdala-dorsolateral prefrontal effective connectivity during explicit emotion processing. European archives of psychiatry and clinical neuroscience, (2019).

75. J. H. Callicott, V. S. Mattay, A. Bertolino, K. Finn, R. Coppola, J. A. Frank, T. E. Goldberg, D. R. Weinberger, Physiological characteristics of capacity constraints in working memory as revealed by functional MRI. Cerebral cortex 9, 20-26 (1999).

76. P. Taurisano, L. A. Antonucci, L. Fazio, A. Rampino, R. Romano, A. Porcelli, R. Masellis, M. Colizzi, T. Quarto, S. Torretta, A. Di Giorgio, G. Pergola, A. Bertolino, G. Blasi, Prefrontal activity during working memory is modulated by the interaction of variation in $\mathrm{CB} 1$ and COX2 coding genes and correlates with frequency of cannabis use. Cortex; a journal devoted to the study of the nervous system and behavior 81, 231-238 (2016).

77. A. Lombardi, C. Guaragnella, N. Amoroso, A. Monaco, L. Fazio, P. Taurisano, G. Pergola, G. Blasi, A. Bertolino, R. Bellotti, S. Tangaro, Modelling cognitive loads in schizophrenia by means of new functional dynamic indexes. NeuroImage 195, 150-164 (2019).

78. P. Selvaggi, G. Pergola, B. Gelao, P. Di Carlo, M. A. Nettis, G. Amico, L. Fazio, A. Rampino, F. Sambataro, G. Blasi, A. Bertolino, Genetic Variation of a DRD2 Co-expression Network is Associated with Changes in Prefrontal Function After D2 Receptors Stimulation. Cereb Cortex, (2018). 
bioRxiv preprint doi: https://doi.org/10.1101/2020.08.03.230227; this version posted August 3, 2020. The copyright holder for this preprint (which was not certified by peer review) is the author/funder. All rights reserved. No reuse allowed without permission.

79. A. Heinz, D. F. Braus, M. N. Smolka, J. Wrase, I. Puls, D. Hermann, S. Klein, S. M. Grusser, H. Flor, G. Schumann, K. Mann, C. Buchel, Amygdala-prefrontal coupling depends on a genetic variation of the serotonin transporter. Nature neuroscience 8, 20-21 (2005). 
PRS $_{\text {miR-137 }}$
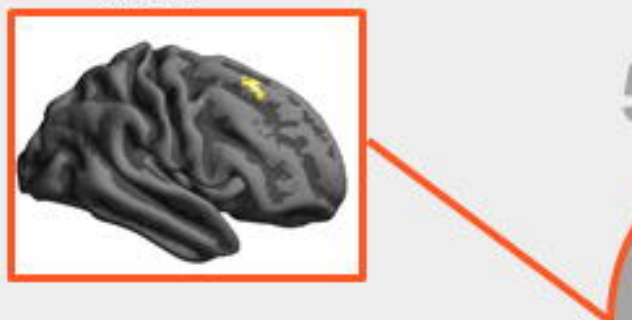

$\mathrm{PCl}_{\text {miR-137 }}$

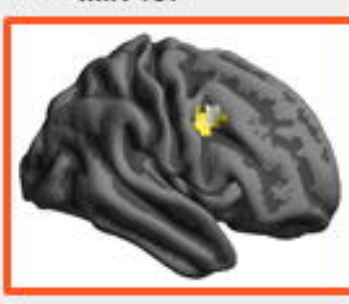

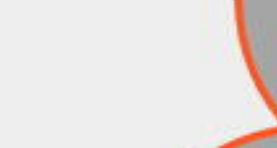

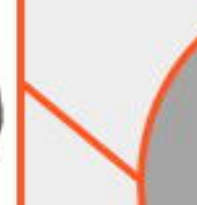

$\mathrm{PCl}_{\text {miR-137 }}$

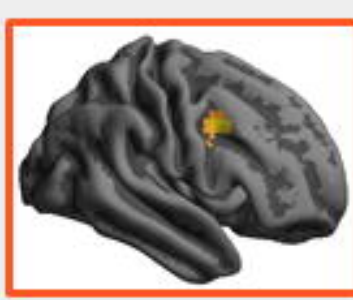

\section{Emotion}

Processing

Discovery set:

$$
\mathrm{N}=222
$$

Replication set:

$\mathrm{N}=136$

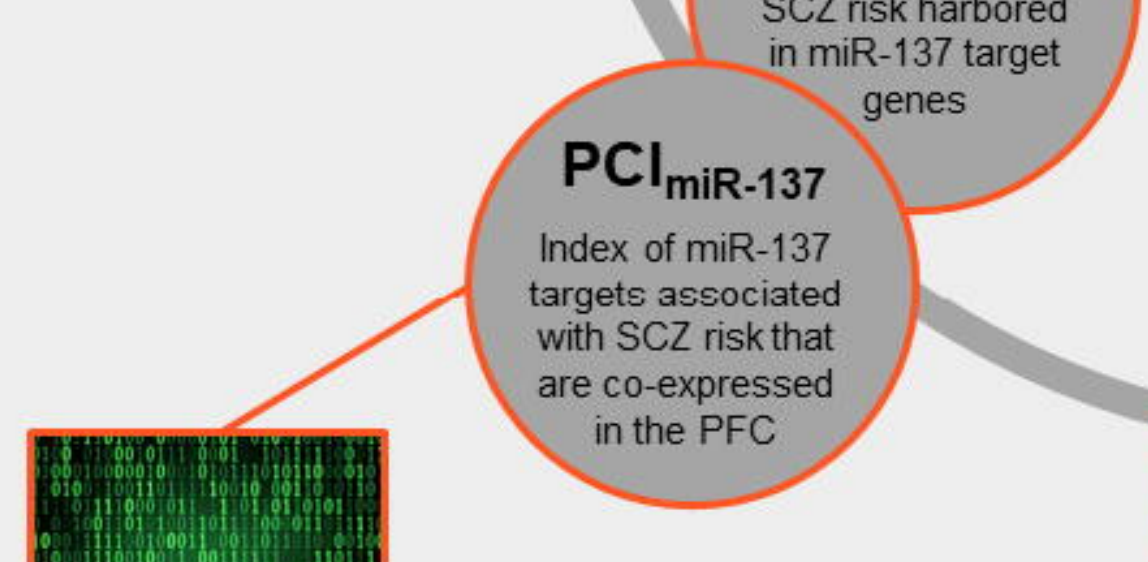

$\mathrm{PCl}_{\text {miR-137 }}$ and $\mathrm{PRS}_{\text {miR-137 }}$ were tested as predictors of both WM and EP activity in the PFC measured with fMRI
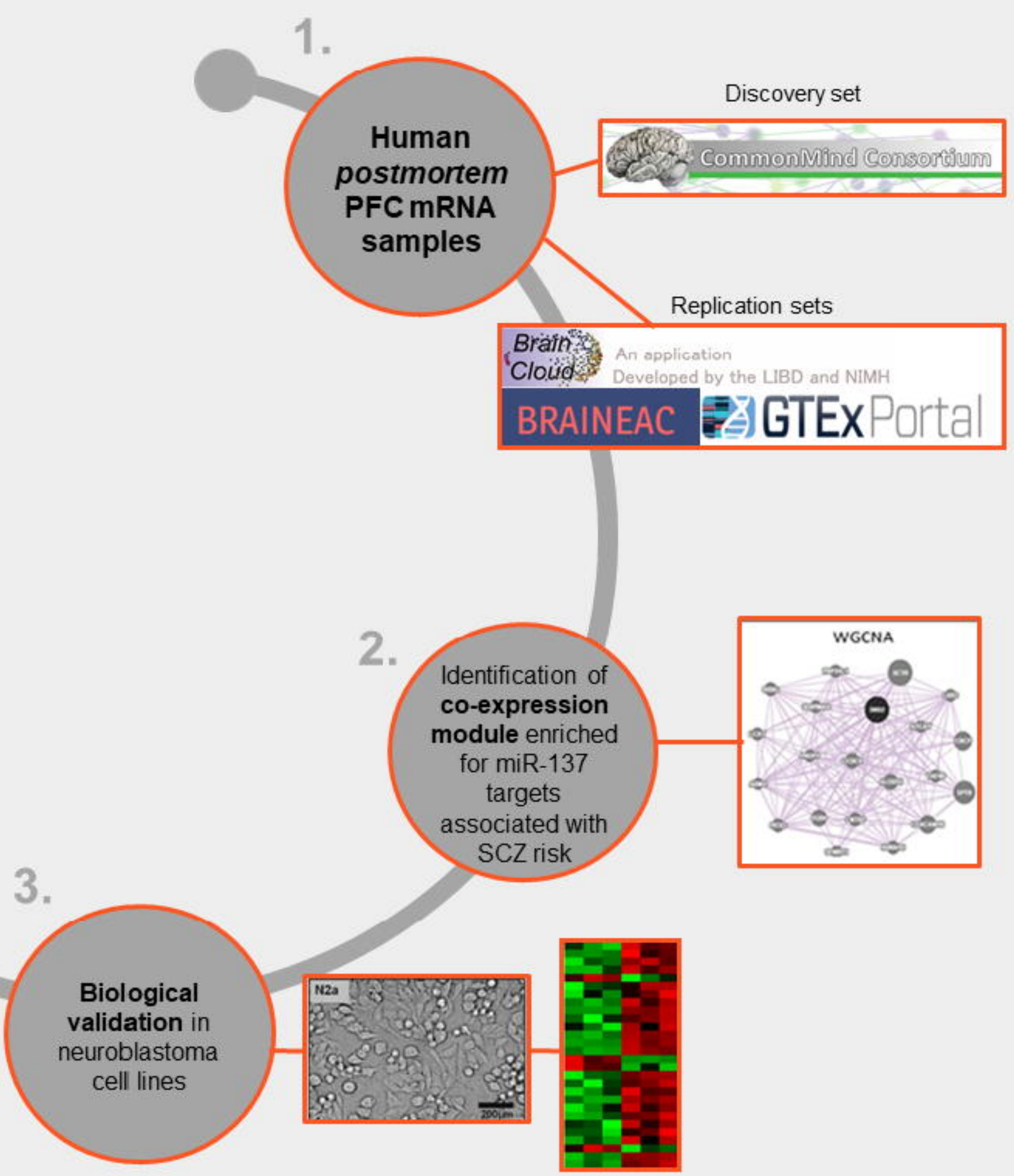

Investigation of causal relationship between miR-137 and differential gene expression in the module 
$\square$ Darkorange $\square$ mir__137 $\square$ PGC

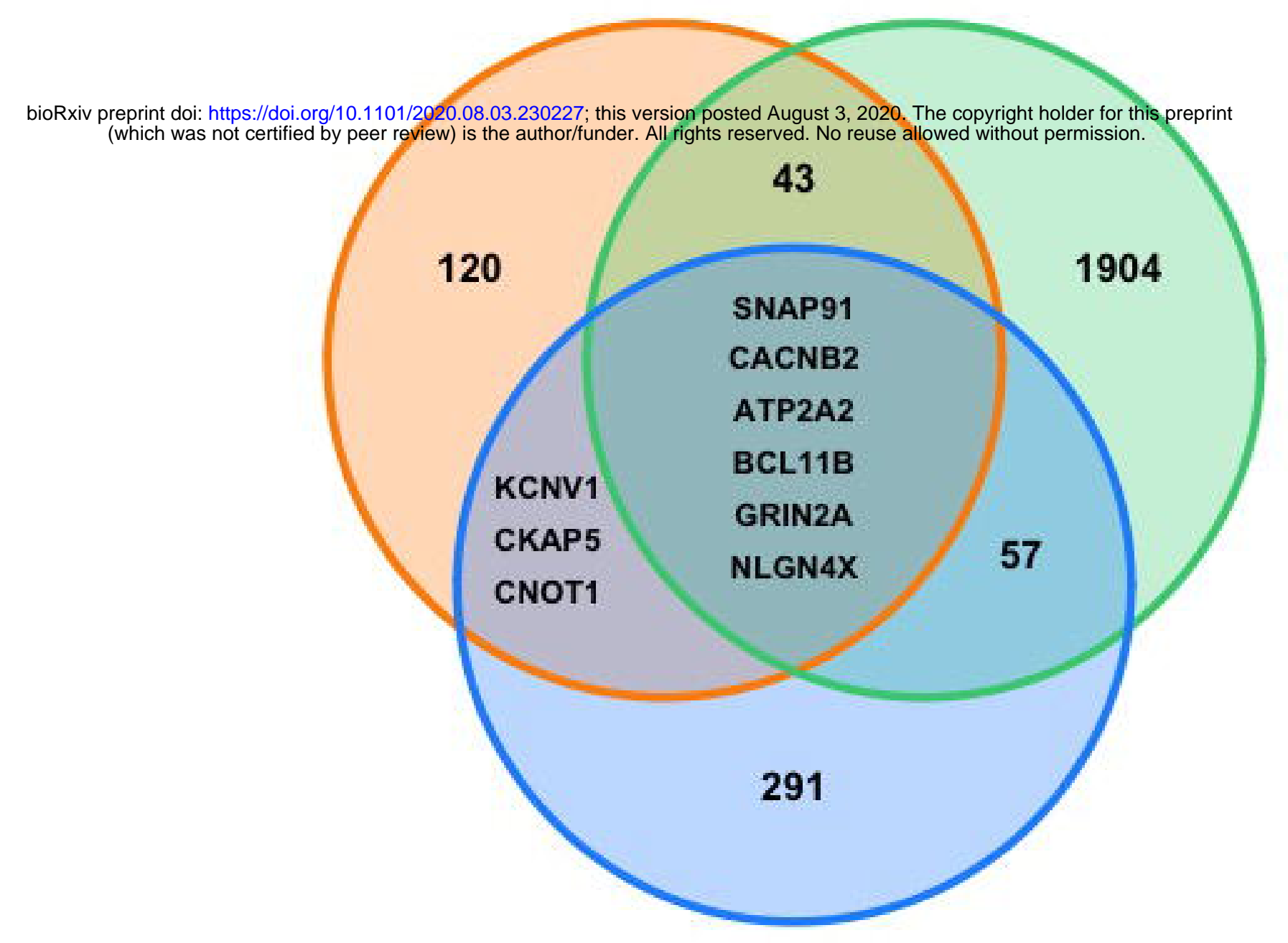

C

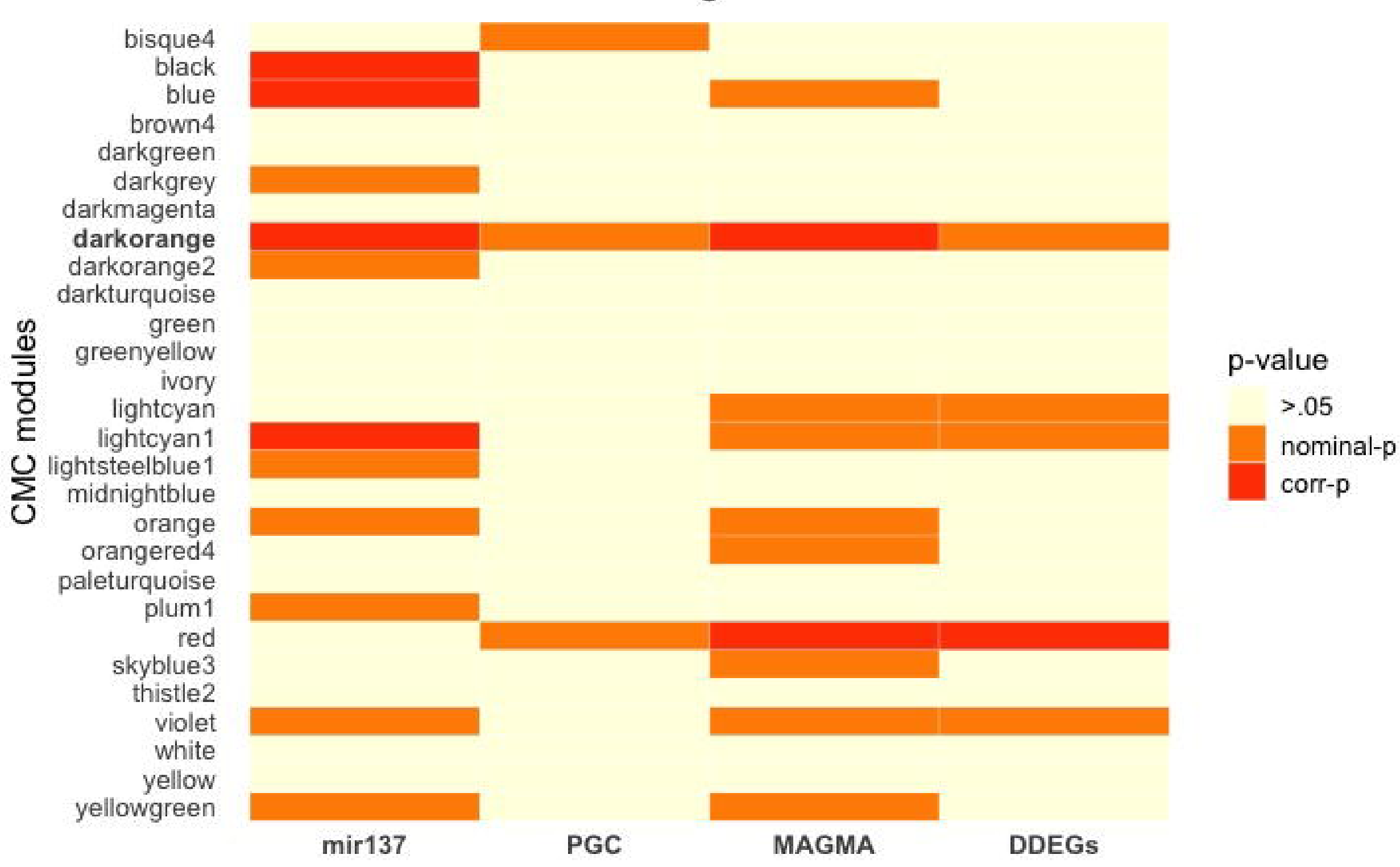

Darkorange gene ontology enrichmen

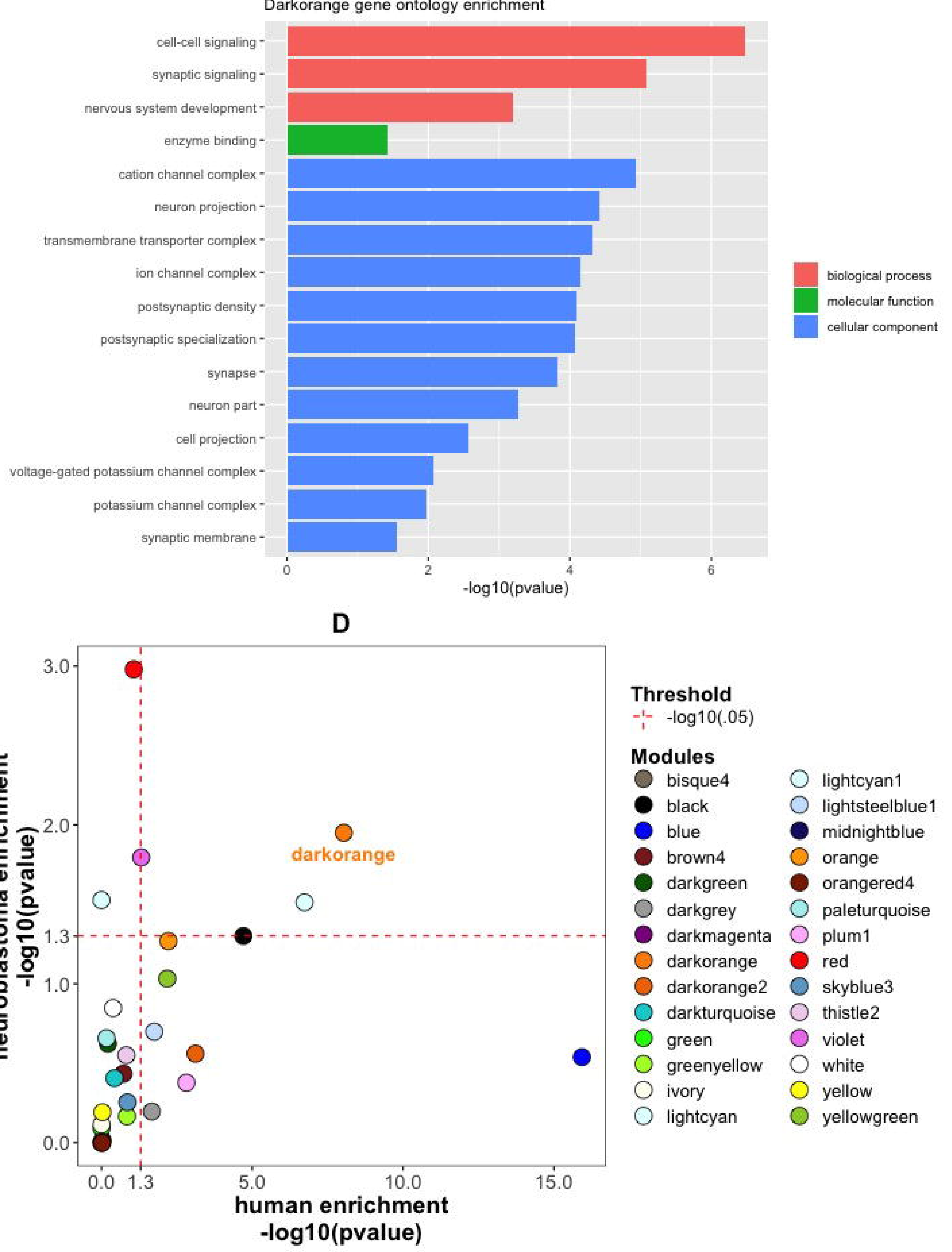




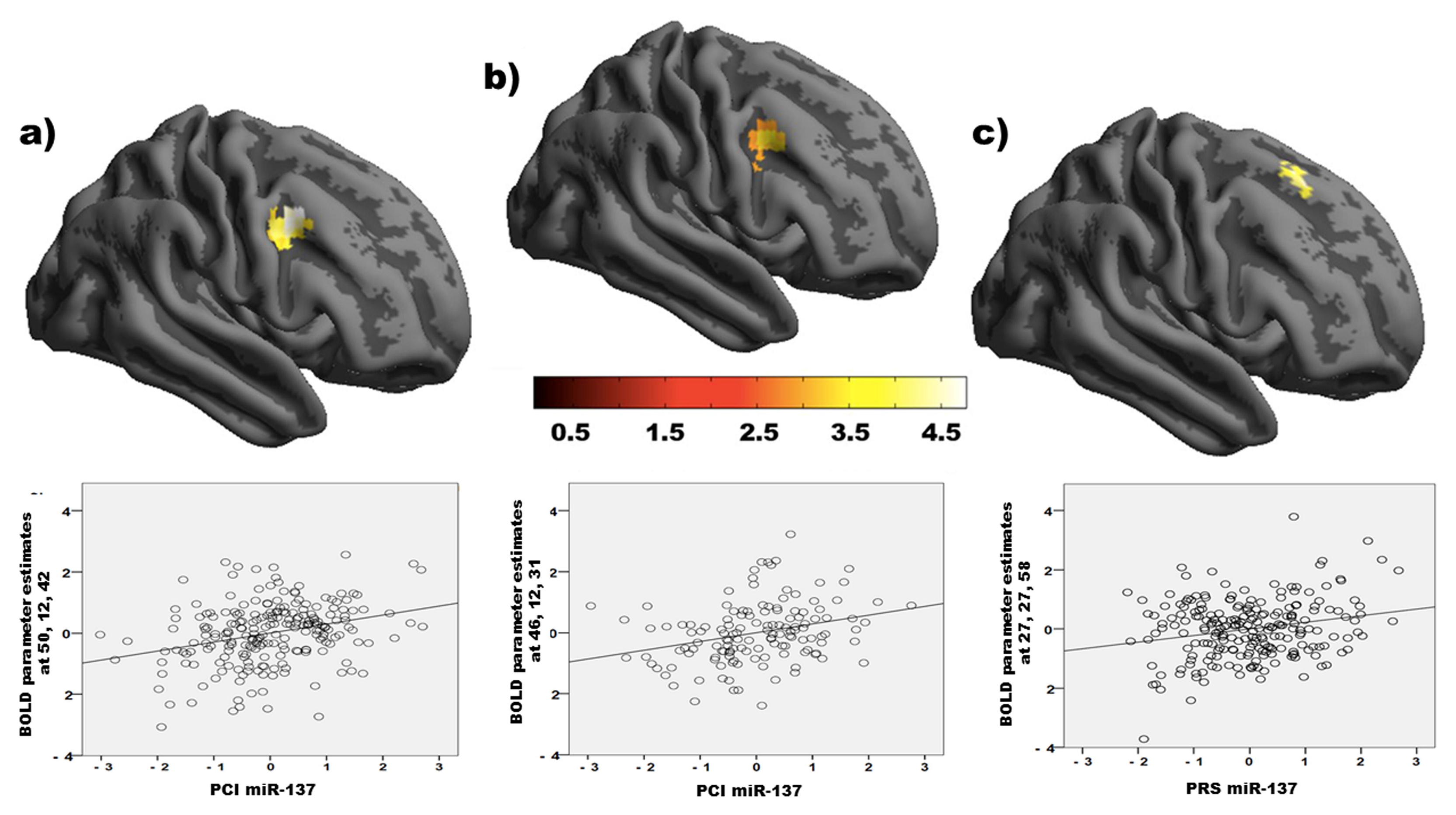

